\title{
Metallicity of solar-type stars with debris discs and planets ${ }^{\star}, \star \star$
}

\author{
J. Maldonado ${ }^{1}$, C. Eiroa ${ }^{1}$, E. Villaver ${ }^{1}$, B. Montesinos ${ }^{2}$, and A. Mora ${ }^{3}$ \\ 1 Universidad Autónoma de Madrid, Dpto. Física Teórica, Módulo 15, Facultad de Ciencias, Campus de Cantoblanco, \\ 28049 Madrid, Spain \\ e-mail: jesus.maldonado@uam.es \\ 2 Centro de Astrobiología (INTA-CSIC), LAEFF Campus, European Space Astronomy Center (ESAC), PO Box 78, \\ 28691 Villanueva de la Cañada, Madrid, Spain \\ 3 ESA-ESAC Gaia SOC, PO Box 78, 28691 Villanueva de la Cañada, Madrid, Spain
}

Received 10 January 2012 / Accepted 6 February 2012

\section{ABSTRACT}

\begin{abstract}
Context. Around $16 \%$ of the solar-like stars in our neighbourhood show IR-excesses due to dusty debris discs and a fraction of them are known to host planets. Determining whether these stars follow any special trend in their properties is important to understand debris disc and planet formation.

Aims. We aim to determine in a homogeneous way the metallicity of a sample of stars with known debris discs and planets. We attempt to identify trends related to debris discs and planets around solar-type stars.

Methods. Our analysis includes the calculation of the fundamental stellar parameters $T_{\text {eff }}, \log g$, microturbulent velocity, and metallicity by applying the iron ionisation equilibrium conditions to several isolated $\mathrm{Fe}$ I and $\mathrm{Fe}$ II lines. High-resolution échelle spectra $(R \sim 57000)$ from $2,3 \mathrm{~m}$ class telescopes are used. Our derived metallicities are compared with other results in the literature, which finally allows us to extend the stellar samples in a consistent way.

Results. The metallicity distributions of the different stellar samples suggest that there is a transition toward higher metallicities from stars with neither debris discs nor planets to stars hosting giant planets. Stars with debris discs and stars with neither debris nor planets follow a similar metallicity distribution, although the distribution of the first ones might be shifted towards higher metallicities. Stars with debris discs and planets have the same metallicity behaviour as stars hosting planets, irrespective of whether the planets are low-mass or gas giants. In the case of debris discs and giant planets, the planets are usually cool, - semimajor axis larger than $0.1 \mathrm{AU}$ ( 20 out of 22 planets), even $\approx 65 \%$ have semimajor axis larger than $0.5 \mathrm{AU}$. The data also suggest that stars with debris discs and cool giant planets tend to have a low dust luminosity, and are among the less luminous debris discs known. We also find evidence of an anticorrelation between the luminosity of the dust and the planet eccentricity.

Conclusions. Our data show that the presence of planets, not the debris disc, correlates with the stellar metallicity. The results confirm that core-accretion models represent suitable scenarios for debris disc and planet formation. These conclusions are based on a number of stars with discs and planets considerably larger than in previous works, in particular stars hosting low-mass planets and debris discs. Dynamical instabilities produced by eccentric giant planets could explain the suggested dust luminosity trends observed for stars with debris discs and planets.
\end{abstract}

Key words. techniques: spectroscopic - stars: abundances - circumstellar matter - stars: late-type - planetary systems

\section{Introduction}

Understanding the origin and evolution of planetary systems is one of the major goals of modern astrophysics. The unexpected discovery by the IRAS satellite of infrared excesses around main-sequence stars (Aumann et al. 1984) was attributed to the

* Based on observations collected at the Centro Astronómico Hispano Alemán (CAHA) at Calar Alto, operated jointly by the Max-Planck Institut für Astronomie and the Instituto de Astrofísica de Andalucía (CSIC); observations made with the Italian Telescopio Nazionale Galileo (TNG) operated on the island of La Palma by the Fundación Galileo Galilei of the INAF (Istituto Nazionale di Astrofisica); observations made with the Nordic Optical Telescope, operated on the island of La Palma jointly by Denmark, Finland, Iceland, Norway, and Sweden, in the Spanish Observatorio del Roque de los Muchachos of the Instituto de Astrofisica de Canarias; and data obtained from the ESO Science Archive Facility.

$\star \star$ Full Tables 1 and 5 are only available in electronic form at the CDS via anonymous ftp to cdsarc.u-strasbg.fr (130.79.128.5) or via

http://cdsarc.u-strasbg.fr/viz-bin/qcat?]/A+A/541/A40 presence of faint dusty discs, produced by collisional events within a significant population of invisible left-over planetesimals. The discovery of these so-called debris discs demonstrated that planetesimals are more common than had been previously thought, revealing that the initial steps of planetary formation are ubiquitous (e.g. Backman \& Paresce 1993). This realisation has been complemented in the past 15 years with the detection of more than 700 exoplanets orbiting stars other than the Sun ${ }^{1}$.

More recent studies have found that more than $50 \%$ of solartype stars harbor at least one planet of any mass with a period of up to 100 days, and about $14 \%$ of this type of stars have planetary companions more massive than $50 M_{\oplus}$ with periods shorter than 10 years (Mayor et al. 2011). It is well-established that the percentage of stars hosting gas-giant planets increases with the metal content, up to $25 \%$ for stars with metallicities higher than +0.30 dex (e.g. Santos et al. 2004; Fischer \& Valenti 2005). On the other hand, stars that host less massive planets, Neptunelike or super Earth-like planets $\left(M_{\mathrm{p}}<30 M_{\oplus}\right)$, do not tend to

$\overline{1 \text { http://exoplanet.eu/ }}$ 
be metal-rich (Ghezzi et al. 2010b; Mayor et al. 2011; Sousa et al. 2011, and references therein). In terms of the metallicity of evolved stars (late-type subgiants and red giants) hosting planets, previous results have been based on the analysis of small and inhomogeneous samples that even produce contradictory results, while these stars are metal-poor in the cases of Pasquini et al. (2007) and Ghezzi et al. (2010a), they show metal enrichment according to Hekker \& Meléndez (2007).

Debris discs are, strictly speaking, signatures of planetesimal systems. About $16 \%$ of the main-sequence solar-like (spectral types F5-K5) stars are known to show an excess at $70 \mu \mathrm{m}$ (e.g. Trilling et al. 2008). If planetesimals were the building blocks of planets and, at the same time, the raw material from which debris discs form, their host stars might be expected to have similar properties. However, the incidence of debris discs is no higher around planet-host stars than around stars without detected planets (Kóspál et al. 2009), and several works do not find any correlation between the presence of a debris disc and the metallicity, or any other characteristic, of the stars with planets (e.g. Beichman et al. 2005; Chavero et al. 2006; Greaves et al. 2006; Moro-Martín et al. 2007; Bryden et al. 2009; Kóspál et al. 2009).

In this paper, we revisit the analysis of the properties of solartype stars hosting planets and/or debris discs. One of the motivations is the increase with respect to previous works of $\sim 50 \%$, in the number of stars with known debris discs and planets, in particular those associated with low-mass planets $\left(M_{\mathrm{p}} \lesssim 30 M_{\oplus}\right)$. We distinguish three different categories: stars with known debris discs but no planets (SWDs hereafter), stars with known debris discs and planets (SWDPs), and stars with known planets but no discs (SWPs). In addition, we consider a comparison sample of stars with no detected planets and no detected debris discs (SWODs). We use our own high-resolution échelle spectra to homogeneously determine some of the stellar properties, particularly metallicity, and in a second step we compare our spectroscopic results with published results. This allows us to increase coherently the stellar samples analysed in this work.

\section{Observations}

\subsection{The stellar sample}

A list of stars with known debris discs, SWDs, was compiled by carefully checking the works of Habing et al. (2001), Spangler et al. (2001), Chen et al. (2005), Beichman et al. (2006), Bryden et al. (2006), Moór et al. (2006), Smith et al. (2006), Moro-Martín et al. (2007), Rhee et al. (2007), Trilling et al. (2007), Trilling et al. (2008), Bryden et al. (2009), Kóspál et al. (2009), Plavchan et al. (2009), Tanner et al. (2009), Koerner et al. (2010), Dodson-Robinson et al. (2011), and Moór et al. (2011). These debris discs were discovered by the IRAS, ISO, and Spitzer telescopes. We compiled a total list of 305 stars, from which we retained for study only the solar-type stars (HIPPARcos spectral type between F5 and K2-K3), leading to a total of 136 stars. Most of the debris discs around these stars were detected at Spitzer-MIPS $70 \mu \mathrm{m}$, with fractional dust luminosities of the order of $10^{-5}$ and higher (Trilling et al. 2008).

To build the comparison sample of stars without discs (SWODs), we selected from the aforementioned works stars in which IR-excesses were not found at 24 and $70 \mu \mathrm{m}$ by Spitzer. As before, only solar-type stars were considered, leading to 150 stars. Since Spitzer is limited up to fractional luminosities of $L_{\text {dust }} / L_{\star} \gtrsim 10^{-5}$, we cannot rule out the possibility that some of these stars have fainter discs.
Table 1. The SWD and SWOD samples.

\begin{tabular}{cccccccc}
\hline \hline HIP & HD & SpType & $V$ & $\begin{array}{c}\text { Distance } \\
(\mathrm{pc})\end{array}$ & $\begin{array}{c}\log (\mathrm{Age}) \\
(\mathrm{yr})\end{array}$ & $\begin{array}{c}{[\mathrm{Fe} / \mathrm{H}]} \\
(\mathrm{dex})\end{array}$ & Ref. \\
\hline \multicolumn{7}{c}{ Stars with known debris discs } \\
171 & 224930 & G3V & 5.80 & 12.17 & 9.60 & $-0.72^{a}$ & 13 \\
490 & 105 & G0V & 7.51 & 39.39 & 8.34 & $-0.03^{b}$ & 2 \\
544 & 166 & K0V & 6.07 & 13.67 & 9.16 & $0.15^{a}$ & 5 \\
682 & 377 & G2V & 7.59 & 39.08 & 8.34 & $0.12^{b}$ & 6 \\
1481 & 1466 & F8/G0 & 7.46 & 41.55 & 8.34 & $-0.22^{c}$ & 7 \\
1598 & 1562 & G0 & 6.97 & 24.80 & 9.79 & $-0.32^{a}$ & 13 \\
1599 & 1581 & F9V & 4.23 & 8.59 & 9.58 & $-0.29^{a}$ & 9 \\
2843 & 3296 & F5 & 6.72 & 45.05 & & $0.02^{c}$ & 9 \\
\hline
\end{tabular}

Notes. Only the first eight lines of the SWD sample and the references are presented here; the full version of the table is available at the CDS. ${ }^{(a)}$ This work; ${ }^{(b)}$ Valenti \& Fischer (2005); ${ }^{(c)}$ Nordström et al. (2004); (b) and $(c)$ values are set into our metallicity scale as described in Sect. 3.2.

References. (1) Habing et al. (2001); (2) Spangler et al. (2001); (3) Chen et al. (2005); (4) Beichman et al. (2006); (5) Bryden et al. (2006); (6) Moór et al. (2006); (7) Smith et al. (2006); (8) Rhee et al. (2007); (9) Trilling et al. (2008); (10) Kóspál et al. (2009); (11) Plavchan et al. (2009); (12) Tanner et al. (2009); (13) Koerner et al. (2010); (14) Moór et al. (2011).

To avoid the effects of planets, planet-hosting stars in both the SWD and SWOD samples were removed, after checking the Extrasolar Planets Encyclopedia ${ }^{2}$. The final number of stars in the SWDs sample is 107: 49 F-type stars, 37 G-type stars, and 21 K-type stars. The SWODs sample contains 145 stars: 62 F-type stars, 65 G-type stars, and 18 K-type stars. Table 1 lists the stars in the SWD and SWOD samples, their properties, and references to debris disc detection.

\subsection{Possible biases}

Metallicity reflects the enrichment history of the ISM (see e.g. Timmes et al. 1995). It is, therefore, important to determine whether the SWD and SWOD samples have randomly selected stellar hosts in terms of age and distance, which are the parameters most likely to affect the metal content of a star. In this respect, we compared the distances and stellar ages of both samples; the results are given in Table 2. Distances are from the updated HipParcos parallaxes (van Leeuwen 2007) and stellar ages were computed from the $\log R_{\mathrm{HK}}^{\prime}$ values given by Maldonado et al. (2010) or from the literature if no value was available in that work. The relationship provided by Mamajek \& Hillenbrand (2008, Eq. (3)) was used to compute the ages. This relationship has an accuracy of $15-20 \%$ for young stars, i.e. younger than $0.5 \mathrm{Gyr}$, and at older age, uncertainties can grow by up to $60 \%$. The age distributions are shown in Fig. 1.

We found a difference between the two samples in terms of the age, with the SWDs containing $15 \%$ more stars younger than 500 Myr. Type II and Type Ia supernova (SNe) are the two sources of Fe production, each operating on different timescales and accounting for very different amounts of the total Fe injected into the ISM. While Type Ia SNe are the major producers of $\mathrm{Fe}$ in galaxies (see e.g. Matteucci \& Greggio 1986), their injection timescales are, according to the most recent estimates, longer than 1 Gyr (Matteucci et al. 2009). In the solar neighbourhood, this 1 Gyr timescale, although uncertain, is the time

2 http://exoplanet.eu/ 
Table 2. Comparison between the properties of the SWDs and the SWODs samples.

\begin{tabular}{lccccccc}
\hline \hline & \multicolumn{3}{c}{ SWDs } & & \multicolumn{3}{c}{ SWODs } \\
\cline { 2 - 4 } \cline { 6 - 7 } \cline { 6 - 7 } & Range & Mean & Median & Range & Mean & Median \\
\hline Distance (pc) & $3.6 / 134$ & 32.0 & 24.6 & & $5.8 / 53$ & 24.1 & 20.6 \\
$\log [$ Age $(\mathrm{yr})]$ & $7.2 / 9.9$ & 9.0 & 9.0 & & $7.6 / 9.9$ & 9.2 & 9.6 \\
\hline SpType (\%) & $45.8(\mathrm{~F}) ; 34.6(\mathrm{G}) ; 19.6(\mathrm{~K})$ & & $42.8(\mathrm{~F}) ; 44.8(\mathrm{G}) ; 12.4(\mathrm{~K})$ \\
\hline
\end{tabular}

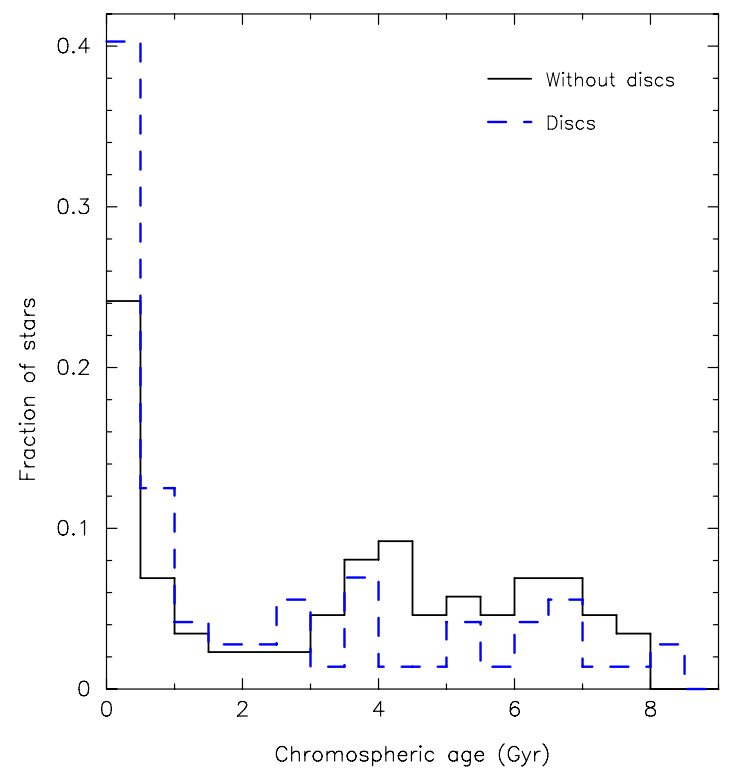

Fig. 1. Age distribution for stars in the SWOD (continuous-black line) and the SWD (dotted-blue line) samples.

at which the Fe production from SNe Ia starts to become important (Matteucci \& Recchi 2001). On the other hand, Type II $\mathrm{SNe}$ are expected to account for only $30 \%$ of the total yield of Fe (Matteucci \& Greggio 1986) but are expected to do so on a shorter timescale (3-5 Myr). A high rate of local SN type II explosions has been estimated to explain the local bubble (Maíz-Apellániz 2001), namely 20 SN type II explosions within $150 \mathrm{pc}$ of the Sun in the past 11 Myr (Benítez et al. 2002). The youngest stars in the SWD and SWOD samples have ages of $15 \mathrm{Myr}$ and $35 \mathrm{Myr}$, respectively, with a larger number of young SWDs in the first $500 \mathrm{Myr}$ bin. The paucity of SNe type II in the Galaxy (typical rate of $\approx 1 \mathrm{SNe} \mathrm{Myr}^{-1}$ ) and all the stars being at relatively close distances from the Sun (less than $130 \mathrm{pc}$ ) make it very unlikely that the two samples have experienced different enrichment histories. We have, however, explored this possibility in Fig. 2, where we plot the metallicity versus age (Sect. 3) for the two samples. As we can see, the SWDs and SWODs have similar behaviours. Young stars in the SWDs sample do not seem to have higher metallicities, so we can rule out a possible chemical evolution in the SWD sample.

We have also checked whether there is a difference between the SWD and SWOD samples in terms of distance that might affect their metallicity distributions. After all, the SWD sample contains stars out to a larger volume than that of the SWODs and could possibly include stars with a different chemical evolution. Garnett \& Kobulnicky (2000) studied the scatter in the age-metallicity relation for $\mathrm{F}$ and $\mathrm{G}$ dwarf stars in the solar neighbourhood up to $80 \mathrm{pc}$, and found that their stars at distances $30-80$ pc from the Sun are more metal-poor than those

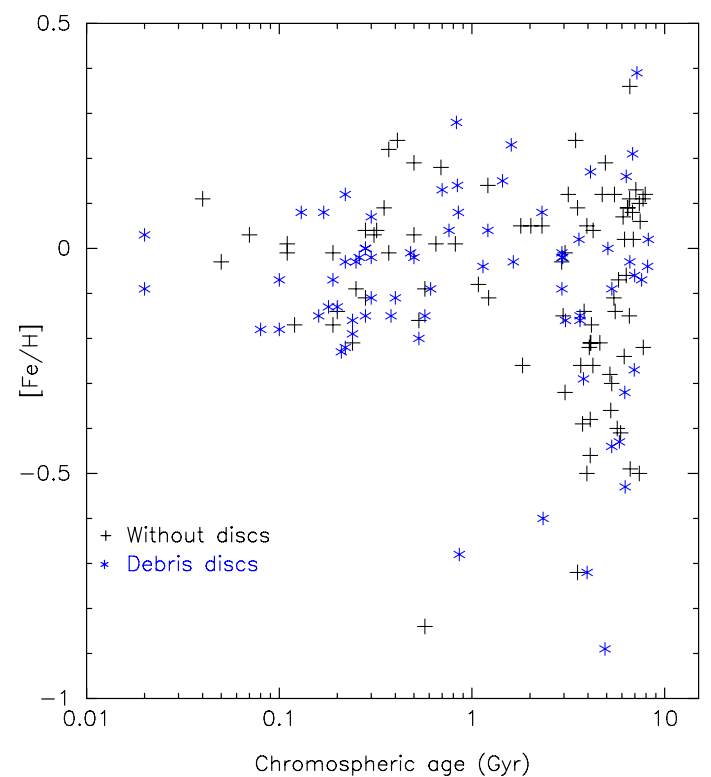

Fig. 2. $[\mathrm{Fe} / \mathrm{H}]$ versus age for the stars in the SWOD (black crosses) and in the SWD (blue asterisks) samples.

within 30 pc. Garnett \& Kobulnicky (2000) attributed this difference to the possible consequence of a selection bias in the analysed sample.

We certainly cover the same volume of stars in our homogeneous SWD and SWOD subsamples (see Sect. 3.1), since they are located within $25 \mathrm{pc}$ of the Sun (Maldonado et al. 2010). We do not find any chemical distinction between these two subsamples. If in the full sample (see Sect. 3.3) we had a selection bias due to the larger distance of the SWODs, we would expect its metallicity distribution to show a larger dispersion owing to a possible contamination by stars not born in the solar neighbourhood. We have the opposite case, where the full samples of both SWODs and SWDs have a smaller dispersion than the volume-limited homogeneous SWDs and SWODs subsamples (see Fig. 3).

In short, we believe that we have a randomly selected sample of stars in terms of their chemical history, although the SWD and SWOD samples show some differences in age and distance.

\subsection{Spectroscopic observations}

The high-resolution spectra used in this work are the same as in Maldonado et al. (2010), where a complete description of the observing runs and the reduction procedure can be found. In brief, the data were taken with the following spectrographs and telescopes: i) FOCES (Pfeiffer et al. 1998) at the $2.2 \mathrm{~m}$ telescope of the Calar Alto observatory (Almería, Spain); ii) SARG (Gratton et al. 2001) at the TNG, La Palma (Canary Islands, Spain); and iii) FIES (Frandsen \& Lindberg 1999) at the NOT, La Palma. We also used additional spectra from the public library " $\mathrm{S}{ }^{4} \mathrm{~N}$ " (Allende Prieto et al. 2004), which contains spectra taken with the 2dcoudé spectrograph at McDonald Observatory and the FEROS instrument at the ESO $1.52 \mathrm{~m}$ telescope in La Silla, and from the ESO/ST-ECF Science Archive Facility ${ }^{3}$ (specifically FEROS spectra). Table 3 lists the spectral range and resolving power of each of the spectrographs. The number of stars covered by these spectra are $35(33 \%)$ and $58(40 \%)$ for the SWD

${ }^{3}$ http://archive.eso.org/cms/ 
Table 3. Properties of the different spectrographs used in this work.

\begin{tabular}{lcc}
\hline \hline Spectrograph & Spectral range $(\AA)$ & Resolving power \\
\hline FOCES & $3470-10700$ & 57000 \\
SARG & $5500-10100$ & 57000 \\
FIES & $3640-7360$ & 67000 \\
FEROS & $3500-9200$ & 42000 \\
Mc Donald & $3400-10900$ & 60000 \\
\hline
\end{tabular}

Table 4. Fe I and Fe II lines used to compute abundances.

\begin{tabular}{lcccc}
\hline \hline & Fe I & & Fe II \\
\cline { 1 - 2 } \cline { 5 - 5 } 4389.25 & 6173.34 & 6699.14 & & 4576.34 \\
4445.48 & 6180.21 & 6739.52 & & 4620.52 \\
5225.53 & 6200.32 & 6750.16 & & 4656.98 \\
5247.06 & 6219.29 & 6752.71 & & 5234.63 \\
5250.22 & 6232.65 & 6793.27 & & 5264.79 \\
5326.15 & 6240.65 & 6804.00 & & 5414.08 \\
5412.79 & 6265.14 & 6804.28 & & 5525.13 \\
5491.84 & 6271.28 & 6837.01 & & 6432.68 \\
5600.23 & 6280.62 & 6854.83 & & 6516.08 \\
5661.35 & 6297.80 & 6945.21 & & 7222.40 \\
5696.09 & 6311.51 & 6971.94 & 7224.46 \\
5701.55 & 6322.69 & 6978.86 & 7515.84 \\
5705.47 & 6353.84 & 7112.17 & & 7711.73 \\
5778.46 & 6481.88 & 7401.69 & \\
5784.66 & 6498.95 & 7723.21 & \\
5855.08 & 6518.37 & 7912.87 & \\
5909.98 & 6574.23 & 8075.16 & \\
5956.70 & 6581.21 & 8204.11 & \\
6082.72 & 6593.88 & 8293.52 & \\
6120.26 & 6609.12 & 8365.64 & \\
6137.00 & 6625.03 & & \\
6151.62 & 6667.72 & & \\
\hline
\end{tabular}

Notes. Wavelengths are given in Angstroms ( $\mathrm{A})$.

and SWOD samples, respectively. Thus, we consider additional data from the literature to analyse the whole set of stars in both samples (see Sect. 3.3).

\subsection{Analysis}

The stellar parameters $T_{\text {eff }}, \log g$, microturbulent velocity $\left(\xi_{t}\right)$, and $[\mathrm{Fe} / \mathrm{H}]$, are determined using the code TGV developed by Takeda et al. (2002), which is based on iron-ionisation equilibrium conditions, a methodology that is widely applied to solarlike stars (spectral types F5/K2-K3). Iron abundances are computed for a well-defined set of Fe I and Fe II lines. Basically, the stellar parameters are adjusted until: i) no dependence is found between the abundances derived from $\mathrm{Fe}$ I lines and the lower excitation potential of the lines; ii) no dependence is found between the abundances derived from the Fe I lines and their equivalent widths; and iii) the derived mean Fe I and Fe II abundances are the same. We list the lines used in Table 4. We are aware that ideally all our targets should have been observed with the same spectrograph using the same configuration. Nevertheless, all the spectra used here have a similar resolution, and cover enough $\mathrm{Fe}$ lines to provide a high-quality metallicity determination. Only for the SARG spectra is the number of Fe II lines slightly lower (6 out of 13, begining in the $6432.68 \AA$, line).
Equivalent widths are obtained by fitting the lines with a Gaussian profile using the IRAF ${ }^{4}$ task splot. Stars with significant rotational velocities, $v \sin i$, have lines affected by blending, complicating the application of this method. Stars with $v \sin i \gtrsim$ $15-20 \mathrm{~km} \mathrm{~s}^{-1}$ typically do not have enough isolated lines to obtain accurate parameters. This has a small impact on our estimates since we consider stars with spectral types F5 or later, with typical $v \sin i$ values in the range $3-9 \mathrm{~km} \mathrm{~s}^{-1}$ (Martínez-Arnáiz et al. 2010). The estimated stellar parameters and iron abundances are given in Table 5.

\section{Results}

\subsection{Homogeneous analysis}

In a first step, we consider the 35 SWDs and 58 SWODs whose metallicities were estimated directly in this work. The stars in these homogeneous samples are listed in Table 5, and are marked in Col. 7 of Table 1 as well. Figure 3 (left panel) shows the normalized distribution of these stars. Both distributions are very similar. The metallicity distribution of the SWOD sample spreads over a large range containing both metal-poor and metalrich stars, from -1.12 to 0.36 dex. The mean metallicity of the distribution is -0.09 dex with an RMS dispersion of 0.27 dex. The distribution of the SWDs spans a slightly narrower range, from -0.89 to 0.35 dex, with a mean value of -0.10 dex and a dispersion of 0.28 dex. Since the mean of a distribution is strongly affected by the presence of outliers, we consider the median as a more representative value. The median values for the SWOD and SWD distributions are -0.01 and -0.02 , respectively. To assess whether both distributions are equal from a statistical point of view, a two-sample Kolmogorov-Smirnov (K-S) test was performed (details about how the K-S test is applied in this paper are given in Appendix A). The maximum difference between the SWD and SWOD cumulative distribution functions is only $\sim 0.11$, while the likelihood that both samples have the same parent distribution is around $94 \%$.

\subsection{Comparison with previous works}

The spectroscopic observations performed by Maldonado et al. (2010) were limited to $25 \mathrm{pc}$ in distance and, therefore, do not cover all SWDs and SWODs in Table 1. Thus, we use the data of Nordström et al. (2004, NO04), Valenti \& Fischer (2005, VF05) and Takeda et al. (2005, TA05) to analyse the full samples. To ensure that we did not introduce any bias resulting from estimates based on different analysis techniques, a comparison between our metallicities and the ones reported in these papers is shown in Fig. 4. Our sample contains 72 stars in common with NO04. Our metallicities are slightly higher, by a factor $\sim 0.07$ dex (in median), than those given by NO04; the differences are largest for stars with positive metallicities. The agreement with VF05 is very good, with no apparent bias for the 64 stars in common; the mean difference is only -0.01 dex with a standard deviation of 0.09 dex. The VF05 metallicities are also higher than the NO04 values by a factor $\sim 0.08$ dex. The agreement with TA05 is excellent, better than \pm 0.10 dex for most of the 49 common stars. The latter result is expected because the same method and lines were used to estimate the metallicity; it can thus be considered a consistency double check.

${ }^{4}$ IRAF is distributed by the National Optical Astronomy Observatory, which is operated by the Association of Universities for Research in Astronomy, Inc., under contract with the National Science Foundation. 

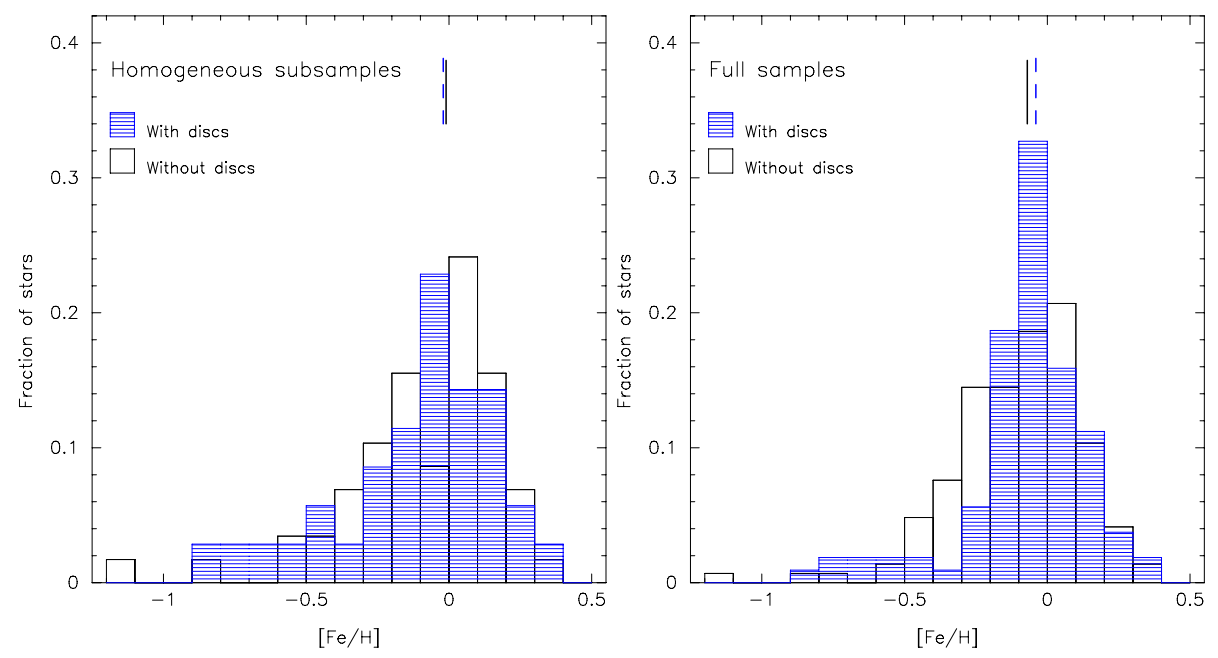

Fig. 3. Normalized metallicity distribution of the stars without debris discs (SWODs, empty histogram), and the stars with debris discs (SWDs, blue histogram shaded at 0 degrees). Median values of the distributions are shown with vertical lines. Left panel: distributions of the stars in the homogeneous sample, i.e., metallicities computed from our own spectra. Right panel: distributions of the full stellar sample (see text).

Table 5. Estimated physical parameters with uncertainties for the stars measured in this work.

\begin{tabular}{|c|c|c|c|c|c|c|c|c|c|c|c|}
\hline HIP & $\begin{array}{l}\text { HD } \\
\text { (2) }\end{array}$ & $\begin{array}{c}\text { Sp Type } \\
\text { (3) }\end{array}$ & $\begin{array}{l}T_{\text {eff }} \\
(\mathrm{K}) \\
(4)\end{array}$ & $\begin{array}{c}\log g \\
\left(\mathrm{~cm} \mathrm{~s}^{-2}\right) \\
(5)\end{array}$ & $\begin{array}{c}\xi_{\mathrm{t}} \\
\left(\mathrm{km} \mathrm{s}^{-1}\right) \\
(6)\end{array}$ & $\begin{array}{c}{[\mathrm{Fe} / \mathrm{H}]} \\
\mathrm{dex} \\
(7)\end{array}$ & $\begin{array}{c}\langle A(\mathrm{Fe} \mathrm{I})\rangle \\
(8)\end{array}$ & (9) & $\begin{array}{c}\langle A(\mathrm{Fe} \text { II })\rangle \\
(10)\end{array}$ & (11) & $\begin{array}{r}\text { Spec. }{ }^{\dagger} \\
(12)\end{array}$ \\
\hline \multicolumn{12}{|c|}{ Stars with known debris discs } \\
\hline 171 & 224930 & G3V & $5491 \pm 31$ & $4.75 \pm 0.12$ & $0.92 \pm 0.40$ & $-0.72 \pm 0.08$ & $6.78 \pm 0.11$ & 52 & $6.78 \pm 0.12$ & 12 & 4 \\
\hline 544 & 166 & K0V & $5575 \pm 51$ & $4.68 \pm 0.14$ & $1.05 \pm 0.25$ & $0.15 \pm 0.05$ & $7.65 \pm 0.06$ & 57 & $7.65 \pm 0.06$ & 13 & 4 \\
\hline 1598 & 1562 & G0 & $5603 \pm 36$ & $4.30 \pm 0.12$ & $0.67 \pm 0.27$ & $-0.32 \pm 0.06$ & $7.18 \pm 0.07$ & 58 & $7.18 \pm 0.09$ & 12 & 1 \\
\hline 1599 & 1581 & F9V & $5809 \pm 39$ & $4.24 \pm 0.12$ & $1.30 \pm 0.30$ & $-0.29 \pm 0.06$ & $7.21 \pm 0.08$ & 59 & $7.22 \pm 0.10$ & 13 & 5 \\
\hline 5336 & 6582 & $\mathrm{G} 5 \mathrm{~V}$ & $5291 \pm 32$ & $4.57 \pm 0.11$ & $0.82 \pm 0.42$ & $-0.89 \pm 0.08$ & $6.61 \pm 0.11$ & 55 & $6.62 \pm 0.12$ & 12 & 4 \\
\hline 5862 & 7570 & F8V & $6111 \pm 35$ & $4.42 \pm 0.10$ & $1.35 \pm 0.17$ & $0.17 \pm 0.03$ & $7.67 \pm 0.04$ & 50 & $7.67 \pm 0.04$ & 13 & 6 \\
\hline 5944 & 7590 & G0 & $5951 \pm 39$ & $4.65 \pm 0.11$ & $1.04 \pm 0.38$ & $-0.02 \pm 0.05$ & $7.48 \pm 0.06$ & 48 & $7.48 \pm 0.08$ & 11 & 1 \\
\hline 7576 & 10008 & G5 & $5293 \pm 68$ & $4.90 \pm 0.19$ & $0.39 \pm 0.45$ & $0.08 \pm 0.06$ & $7.58 \pm 0.07$ & 53 & $7.58 \pm 0.10$ & 9 & 1 \\
\hline
\end{tabular}

Notes. Columns 8 and 10 give the mean iron abundance derived from Fe I and Fe II lines, respectively, while Cols. 9 and 11 give the corresponding number of lines. The rest of the columns are self-explanatory. Only the first eight lines are shown here; the full version of the table is available at the CDS. ${ }^{(\dagger)}$ Spectrograph: (1) CAHA/FOCES; (2) TNG/SARG; (3) NOT/FIES; (4) S N-McD; (5) S ${ }^{4}$ N-FEROS; (6) ESO/FEROS.

\subsection{Full sample}

To set the VF05 and NO04 metallicities on our own metallicity scale, we used the stars in common to obtain a linear transformation (Fig. 4). Where possible, VF05 values were selected because they have been obtained from high-resolution spectra similar to those used in this work. The metallicities in NO04 are based on Strömgren $u v b y \beta$ photometry. The adopted final metallicity values for each star of the SWD and SWOD samples are given in Table 1.

Some statistical diagnostics for the SWD and SWOD full samples are summarised in Table 6. Both samples have similar distributions. The full SWD distribution has a median of -0.04 dex, very close to the value obtained in the homogeneous analysis (-0.02 dex). In the case of the SWOD sample, the full sample has a median of -0.07 dex that, when compared with the value of -0.01 dex for the homogeneous subsample, means a difference of 0.06 dex. We note that 0.06 dex is of the order of the individual uncertainties in metallicity. The SWD and SWOD distributions have a smaller dispersion when we consider the whole sample (Fig. 3, right panel). Using a K-S analysis, we tested the possibility of both distributions to differing within a $98 \%$ confidence level; our results cannot exclude that both samples come from the same parent distribution at this confidence level. Nevertheless, the likelihood that both samples are drawn from the same parent distribution diminishes significantly with respect to the homogeneous sample case (9\%, see Appendix A). An interesting aspect is that there seems to be a "deficit" of stars with discs in the metallicity range $-0.50<[\mathrm{Fe} / \mathrm{H}]<-0.20$. This deficit is not explicit in the homogeneous case (Fig. 3, see also Sect. 4 and Fig. 7).

\subsection{Stars with known debris discs and planets}

At the time of writting 5 , there are, to our knowledge, 29 solartype stars known to host both a debris disc and at least one planet (SWDPs). This figure represents an increase of $\sim 50 \%$ with respect to the most recent works (Bryden et al. 2009; Kóspál et al. 2009) $)^{6}$. These stars are listed in Table 7.

Among the 29 SWDPs, 11 stars host known multiplanet systems, which represents an incidence rate of $38 \%$. Wright et al. (2009) found a rate of $14 \%$ confirmed multiple planetary systems, and it could be $28 \%$ or higher when they include cases with a significant evidence of being multiple ${ }^{7}$. Mayor et al. (2011) found a rate exceeding $70 \%$ among their 24 systems with planets less massive than $30 M_{\oplus}$. In our SWDP sample, there are five stars with low-mass planets in multiple systems, but this might

\footnotetext{
5 December 26, 2011.

${ }^{6}$ For the 22 stars with debris discs and planets given by Kóspál et al. (2009), HD 33636 has a substellar companion that has been retracted as a planet (Bean et al. 2007), GJ 581 is a M star, and HD 137759 is a giant star. In addition, Bryden et al. (2009) listed HD 150706 as hosting a planet and a debris disc, but the planet is not confirmed (http:// exoplanet.eu).

7 See also for comparison http//exoplanet.eu
} 
A\&A 541, A40 (2012)
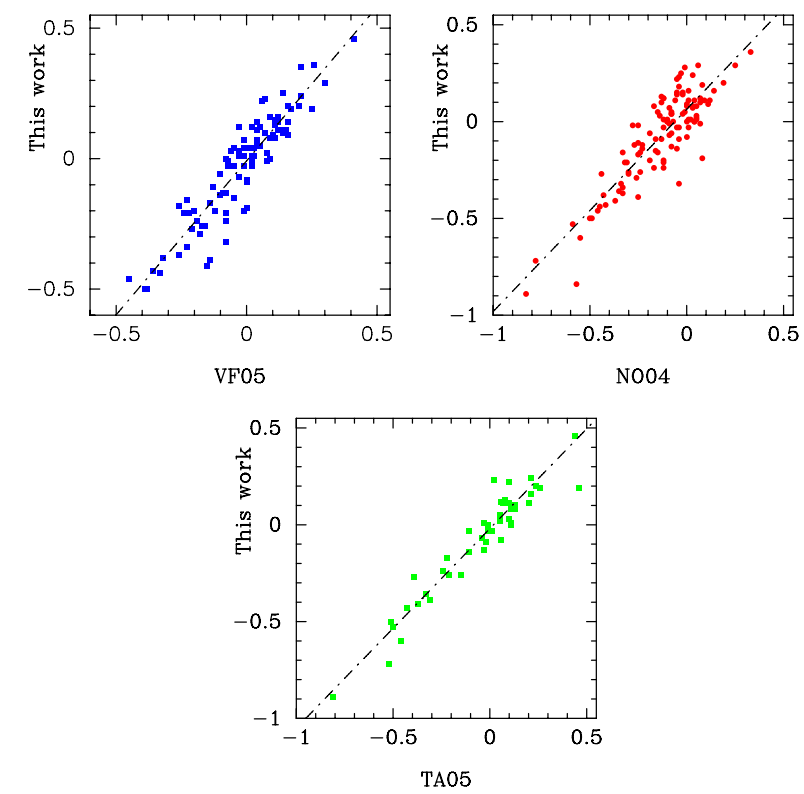

Fig. 4. Comparison between the metallicities from the literature and those obtained in this work. Top left panel: VF05; top right panel: NO04; bottom panel: TA05. Dashed lines represent the best linear fit $(y=m * x+b)$ between our metallicities and those given in the corresponding works. The coefficients are: $m=1.18 \pm 0.05, b=$ $-0.008 \pm 0.008$ for VF05; $m=1.04 \pm 0.05, b=0.064 \pm 0.013$ for N04; and $m=0.99 \pm 0.05, b=-0.017 \pm 0.011$ for TA05.

be a lower limit, as pointed out by Mayor et al. (2011). This suggests that the multiplanet system rate in SWDPs approaches that of the low-mass planet case.

There are 7 of 29 SWDPs that host at least one low-mass planet, $M \lesssim 30 M_{\oplus}$. These stars are HD 1461, HD 20794, HD 38858, HD 45184, HD 69830, 61 Vir (HD 115617), and HD 215152; in all cases, but in HD 1461, their metallicity is $[\mathrm{Fe} / \mathrm{H}] \leq 0.0$, consistent with the metallicity trend for stars with low-mass planets (e.g. Mayor et al. 2011; Sousa et al. 2011).

Wright et al. (2009, Fig. 9) and Currie (2009, Fig. 1) showed that there is an enhanced frequency of close-in gas giant planets with semimajor axes $\lesssim 0.07$ AU (hot Jupiters). Among the 22 SWDPs that are currently known to host only gas-giant planets, HD 46375 is the only star harbouring such a close-in planet, semimajor axis of $0.041 \mathrm{AU}$, while HD 130322 has a hot Jupiter at $0.088 \mathrm{AU}$; five more stars have giant planets with semimajor axes smaller than 0.5 AU (HD 38529, HD 104067, HD 117176, HD 178911B, and HD 192263). On the other hand, the semimajor axes of the low-mass planets are $\lesssim 0.07$ AU in all cases, but in HD 20794 and HD 38858 .

The statistical properties of the SWDP metallicity distribution are shown in Table 6, while Fig. 5 (left) compares the corresponding SWDP histogram with the SWDs. The figure clearly shows the distinct metallicity distributions of both the SWDPs and the SWDs; a K-S test confirms that both distributions differ within a confidence level of $98 \%$ (the likelihood of being the same distribution is $0.7 \%$ ).

Summarizing, although there may be some bias related to the planet detection methods as well as the sensitivity in detecting debris discs, our results suggest that SWDPs i) have higher metallicities than both SWDs and SWODs (see Figs. 5 and 7); ii) they tend to have a higher incidence of multiplanet systems, most likely at a rate close to the one of stars with low-mass planets; iii) many of them host low-mass planets, and iv) in the cases
Table 6. $[\mathrm{Fe} / \mathrm{H}]$ statistics of the stellar samples.

\begin{tabular}{lcccccc}
\hline \hline Sample & Mean & Median & Deviation & Min & Max & $N$ \\
\hline SWODs & -0.09 & -0.07 & 0.22 & -1.12 & 0.36 & 145 \\
SWDs & -0.08 & -0.04 & 0.26 & -1.49 & 0.39 & 107 \\
SWDPs & 0.08 & 0.05 & 0.17 & -0.34 & 0.36 & 29 \\
SWPs & 0.10 & 0.15 & 0.22 & -0.70 & 0.43 & 120 \\
\hline
\end{tabular}

Table 7. Stars with known debris discs and planets.

\begin{tabular}{lccccc}
\hline \hline HIP & HD & Sp Type & $\begin{array}{c}{[\mathrm{Fe} / \mathrm{H}]} \\
(\mathrm{dex})\end{array}$ & Ref. & Planet $^{\star}$ \\
\hline 522 & 142 & $\mathrm{~F} 7 \mathrm{~V}$ & $0.09^{b}$ & $9:$ & $\mathrm{gc}$ \\
1499 & 1461 & $\mathrm{G} 0 \mathrm{~V}$ & $0.18^{b}$ & 11 & $\mathrm{mlh}$ \\
7978 & 10647 & $\mathrm{~F} 8 \mathrm{~V}$ & $-0.09^{a}$ & 3 & $\mathrm{gc}$ \\
14954 & 19994 & $\mathrm{~F} 8 \mathrm{~V}$ & $0.19^{a}$ & 8 & $\mathrm{gc}$ \\
15510 & 20794 & $\mathrm{G} 8 \mathrm{~V}$ & $-0.34^{a}$ & 10 & $\mathrm{mlc}$ \\
16537 & 22049 & $\mathrm{~K} 2 \mathrm{~V}$ & $-0.08^{a}$ & 1 & $\mathrm{gc}$ \\
27253 & 38529 & $\mathrm{G} 4 \mathrm{~V}$ & $0.31^{b}$ & 6 & $\mathrm{mgc}$ \\
27435 & 38858 & $\mathrm{G} 4 \mathrm{~V}$ & $-0.27^{a}$ & 4 & $\mathrm{lc}$ \\
28767 & 40979 & $\mathrm{~F} 8$ & $0.13^{b}$ & $10:$ & $\mathrm{gc}$ \\
30503 & 45184 & $\mathrm{G} 2 \mathrm{~V}$ & $0.03^{b}$ & 11 & $\mathrm{lh}$ \\
31246 & 46375 & $\mathrm{~K} 1 \mathrm{IV}$ & $0.23^{b}$ & $10:$ & $\mathrm{gh}$ \\
32970 & 50499 & $\mathrm{G} 1 \mathrm{~V}$ & $0.29^{b}$ & $10:$ & $\mathrm{gc}$ \\
33212 & 50554 & $\mathrm{~F} 8$ & $-0.09^{b}$ & 8 & $\mathrm{gc}$ \\
$33719^{\sharp}$ & 52265 & $\mathrm{G} 0 \mathrm{~V}$ & $0.18^{b}$ & 8 & $\mathrm{gc}$ \\
40693 & 69830 & $\mathrm{~K} 0 \mathrm{~V}$ & $0.00^{a}$ & 2 & $\mathrm{mlh}$ \\
42282 & 73526 & $\mathrm{G} 6 \mathrm{~V}$ & $0.22^{b}$ & $10:$ & $\mathrm{mgc}$ \\
47007 & 82943 & $\mathrm{G} 0$ & $0.23^{b}$ & 8 & $\mathrm{mgc}$ \\
58451 & 104067 & $\mathrm{~K} 2 \mathrm{~V}$ & $0.04^{b}$ & 11 & $\mathrm{gc}$ \\
61028 & 108874 & $\mathrm{G} 5$ & $0.17^{b}$ & 12 & $\mathrm{mgc}$ \\
64924 & 115617 & $\mathrm{G} 5 \mathrm{~V}$ & $0.00^{a}$ & 8 & $\mathrm{mlh}$ \\
65721 & 117176 & $\mathrm{G} 5 \mathrm{~V}$ & $-0.03^{a}$ & 8 & $\mathrm{gc}$ \\
71395 & 128311 & $\mathrm{~K} 0$ & $0.04^{a}$ & 8 & $\mathrm{mgc}$ \\
72339 & 130322 & $\mathrm{~K} 0 \mathrm{~V}$ & $-0.07^{b}$ & 12 & $\mathrm{gh}$ \\
94075 & $178911 \mathrm{~B}$ & $\mathrm{G} 5$ & $0.29^{b}$ & $10:$ & $\mathrm{gc}$ \\
97546 & 187085 & $\mathrm{G} 0 \mathrm{~V}$ & $0.05^{b}$ & $10:$ & $\mathrm{gc}$ \\
99711 & 192263 & $\mathrm{~K} 2$ & $-0.01^{a}$ & 5 & $\mathrm{gc}$ \\
104903 & 202206 & $\mathrm{G} 6 \mathrm{~V}$ & $0.36^{b}$ & 10 & $\mathrm{mgc}$ \\
112190 & 215152 & $\mathrm{~K} 0$ & $-0.10^{c}$ & 11 & $\mathrm{mlh}$ \\
113044 & 216435 & $\mathrm{G} 3 \mathrm{IV}$ & $0.24^{b}$ & 10 & $\mathrm{gc}$ \\
\hline & & & & &
\end{tabular}

Notes. ${ }^{(a)}$ This work; ${ }^{(b)}$ Valenti \& Fischer (2005), (b) values are set into our metallicity scale as described in Sect. 3.2. ${ }^{\left({ }^{()}\right)}$Metallicity for this star is from Sousa et al. (2008) since no value were found in VF05 or NO04. ${ }^{(\star)} \mathrm{m}=$ multiplanet system; 1 = low-mass planet; $\mathrm{g}=$ gas giant planet; $\mathrm{c}=$ cool planet; $\mathrm{h}=$ hot planet (semimajor axis $\leq 0.1 \mathrm{AU}$, see text). (\#) Spectral type from Montes et al. (2001).

References. (1) Habing et al. (2001); (2) Bryden et al. (2006); (3) Moór et al. (2006); (4) Beichman et al. (2006); (5) Smith et al. (2006); (6) Moro-Martín et al. (2007); (7) Rhee et al. (2007); (8) Trilling et al. (2008); (9) Bryden et al. (2009); (10) Kóspál et al. (2009); (11) Koerner et al. (2010); (12) Dodson-Robinson et al. (2011). The symbol ":" means that non-excess is attributed to the corresponding star in (9) or (10).

with only gas-giant planets, these planets tend to be cool Jupiters (only two out of 22 stars harbour one hot Jupiter).

\subsection{Comparison with stars with giant planets}

Figure 5 (right) shows the metallicity distributions of both SWDPs and SWPs. The SWPs sample contains 120 stars and corresponds to stars hosting exclusively giant planets from Santos et al. (2004), Valenti \& Fischer (2005), Sousa et al. 

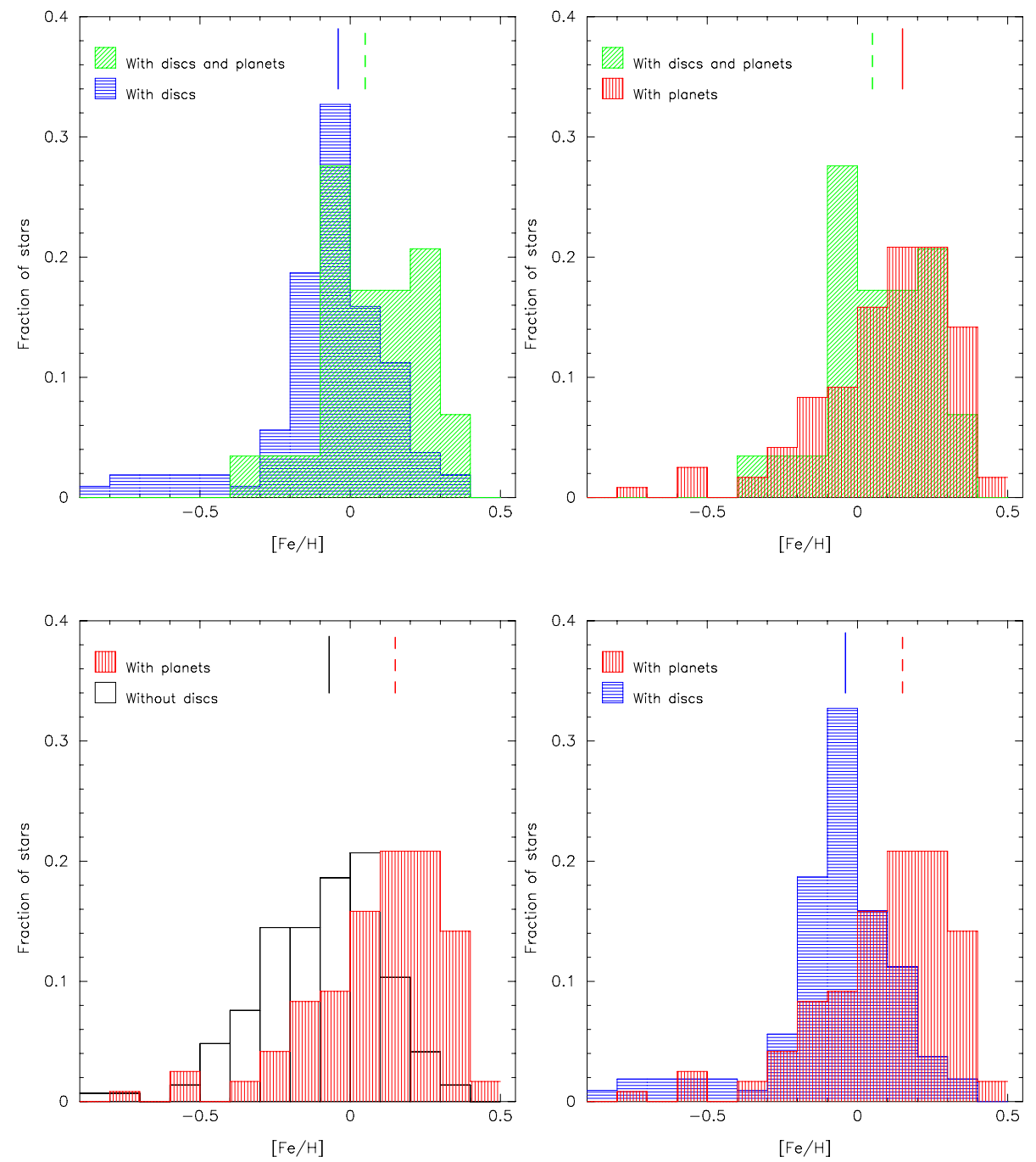

Fig.5. Normalized metallicity distribution of the SWDP sample (light green histogram) versus stars with debris discs (left) and stars with giant planets (right). Median values of the distributions are shown with vertical lines.
Fig. 6. Normalized metallicity distributions of planet host-stars (red histogram) versus stars without debris discs (left) and stars with debris discs $(r i g h t)$. Median values of the distributions are shown with vertical lines.
(2011), and Mayor et al. (2011), where we have removed the stars with retracted or not-confirmed exoplanets. Both histograms clearly show that the stars in the SWP and SWDP samples tend to have high metallicity. The K-S tests cannot rule out that both distributions are the same $(p$-value $=49 \%)$.

With the aim of completeness, Fig. 6 compares the metallicity distribution of the SWPs, with those of the SWODs and SWDs samples, where the well-known trend of SWPs (gas-giant planets) to higher metallicities is clearly reproduced.

\section{Discussion}

The results presented in the previous section suggest that a transition toward higher metallicities occurs from SWODs to SWPs. The cumulative metallicity distributions, presented in Fig. 7, allow us to get an unified overview of the metallicity trends. As pointed out before, the distribution of SWDs is similar to that of SWODs, but there seems to be a deficit of SWDs at low $[\mathrm{Fe} / \mathrm{H}]$, below approximately -0.1 (see also the histogram for the full samples in Fig. 3 (right panel) and the median $[\mathrm{Fe} / \mathrm{H}]$ value in Table 6). The distribution of SWDPs can be clearly distinguished from that of SWDs and is similar to that of SWPs. Thus, planets are clearly the main drivers of the trend in stellar metallicity in SWDPs; this is true for both the low-mass and the giant planets in the SWDP sample. The metallicity distribution of SWPs was divided into hot and cool Jupiters because most of the SWDPs hosting giant planets are associated with cool planets. Figure 7 suggests that the frequency of hot giant planets is lower for low metallicities than the frequency of cool ones. We point out that a similar trend is obtained, when the data refer to all known solartype stars hosting giant planets, i.e., stars with close-in giant planets tend to be more metal-rich.

These trends can be explained by core-accretion models (e.g. Pollack et al. 1996; Ida \& Lin 2004; Hubickyj et al. 2005; Mordasini et al. 2009, 2012), and are consistent with the view that the mass of solids in proto-planetary discs is the main factor controlling the formation of planets and planetesimals (Greaves et al. 2007; Moro-Martín et al. 2007). Thus, the rapid build-up of a core in a metal-rich proto-planetary disc would allow giant planets to form before the dissipation of the gas, while the formation of planetesimals could proceed slowly after the gas dissipation and also in a less metal-rich environment. We note that planetesimals could form regardless of the giant planet formation, and that the timescale for Earth-like planet formation is long and can proceed in a relatively metal-poor environment.

Figure 8 shows the fractional dust luminosity, $L_{\text {dust }} / L_{\star}$, of the SWDs and SWDPs versus the metallicity. The plot distinguishes between low-mass and gas giant planets. Values of $L_{\text {dust }} / L_{\star}$ are taken from the references in Sect. 2.1; we plot the mean value of $L_{\text {dust }} / L_{\star}$ for the stars from Trilling et al. (2008). It is found that the SWDPs as a whole span approximately two orders of magnitude in $L_{\text {dust }} / L_{\star}$ and are well-mixed with SWDs, while 


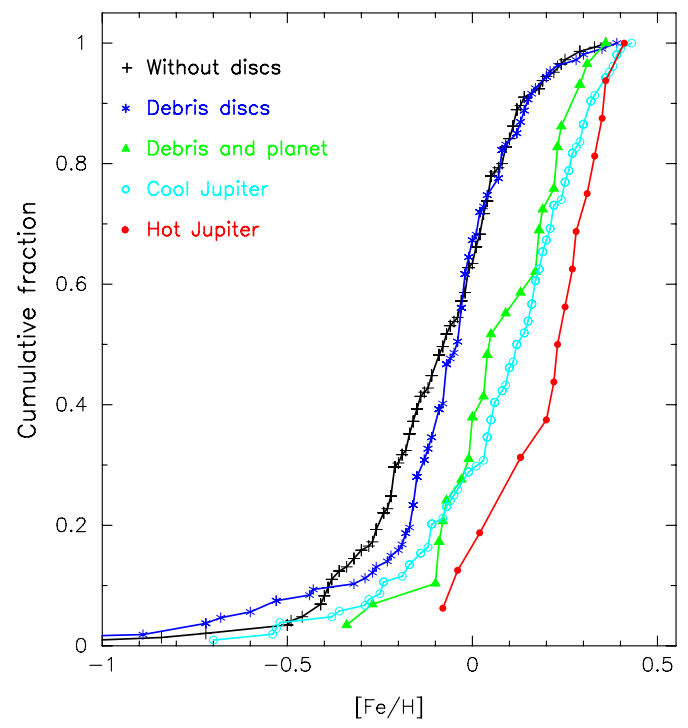

Fig. 7. Histogram of cumulative frequencies for the different samples studied in this work.

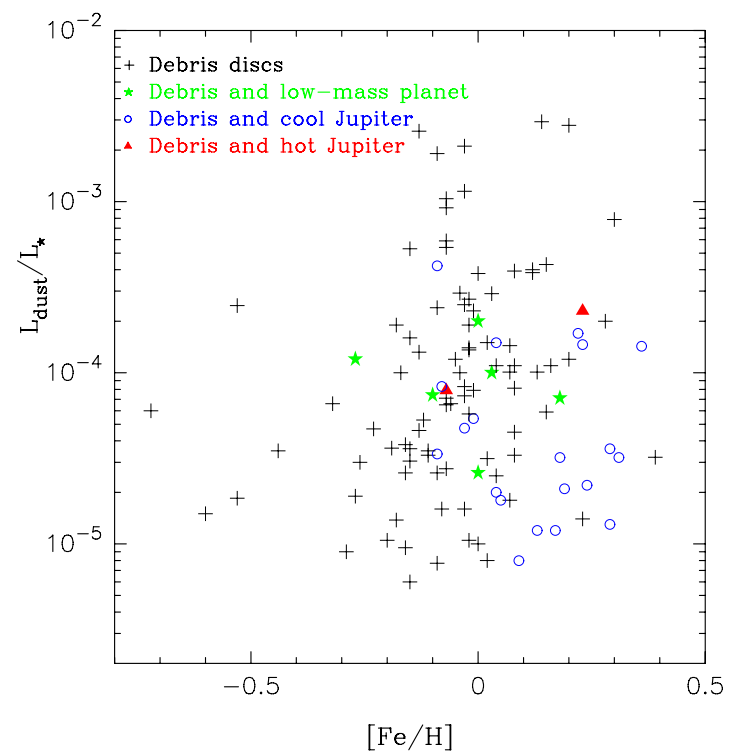

Fig. 8. Fractional dust luminosity, $L_{\text {dust }} / L_{\star}$, versus $[\mathrm{Fe} / \mathrm{H}]$ for those stars hosting a debris discs. Stars are plotted with different symbols and colours depending on the presence/absence of low-mass or cool/hot Jupiter planets.

most of the stars hosting debris discs and cool giant planets tend to have low dust luminosities, $L_{\text {dust }} / L_{\star}<10^{-4}$; more than $50 \%$ of SWDPs of this type are indeed concentrated in the low-dust luminosity/high-metallicity corner of Fig. 8. In addition, there seems to be a trend of larger eccentricities (we take as reference the innermost planet) while the luminosity of the dust decreases, albeit with a large scatter (Fig. 9). Such an anticorrelation may be the result of dynamical instabilities produced by eccentric giant planets, which clear out the inner and outer regions of the planetary discs (Raymond et al. 2011). On the other hand, there is no trend with the semimajor axis of the planet (not shown), although it seems that low-mass planets tend to be predominantly hot but most of the giant planets are cool (Sect. 3.4). Furthermore, while the SWDs span the $\sim 10 \mathrm{Myr}-10$ Gyr range, the SWDPs are mature stars (older than

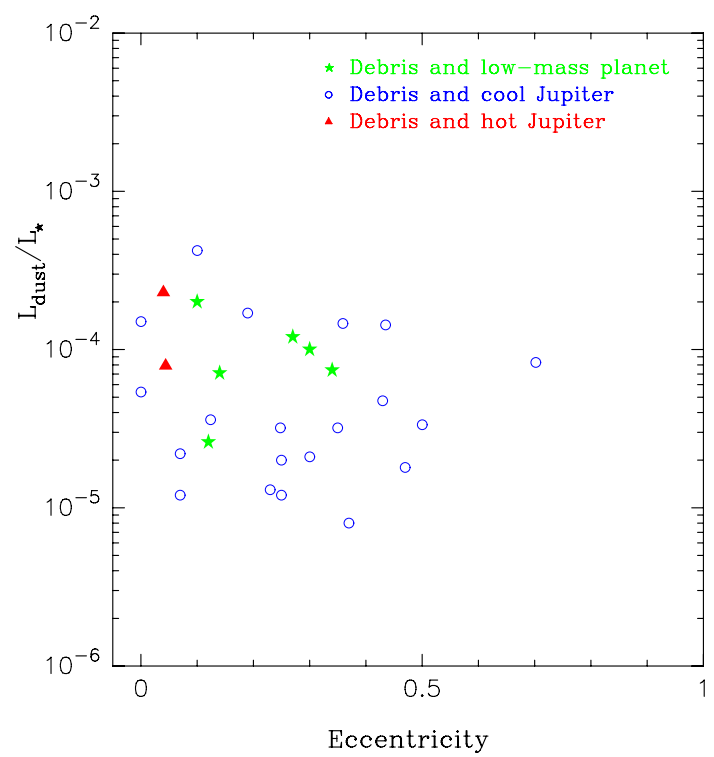

Fig. 9. Fractional dust luminosity, $L_{\text {dust }} / L_{\star}$, versus eccentricity.

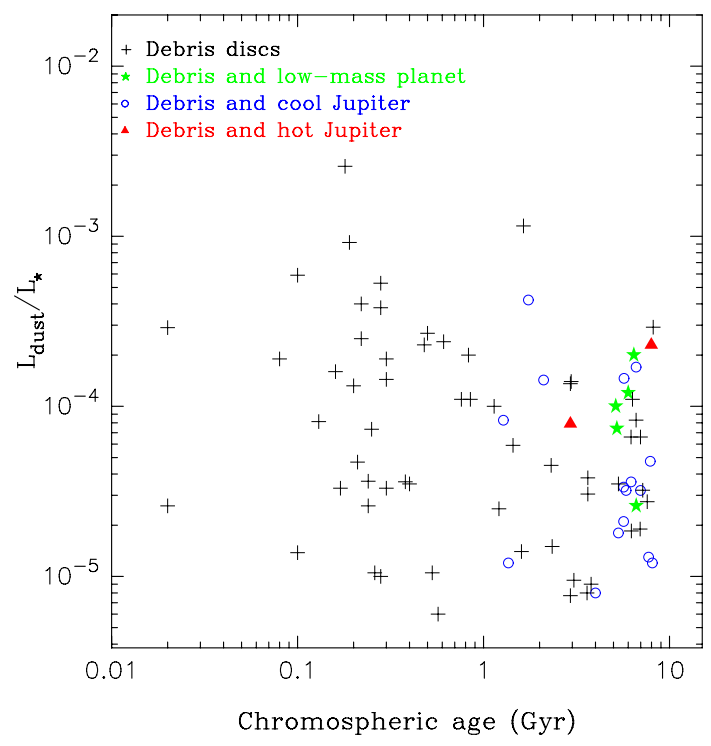

Fig. 10. Fractional dust luminosity, $L_{\text {dust }} / L_{\star}$, versus stellar age.

1 Gyr), although low-mass planet host stars tend to group at old ages, $>5 \mathrm{Gyr}$, and the cool giant-planet stars span a larger range of 1-10 Gyr (Fig. 10). This age behaviour reflects a bias introduced by current planet-detection techniques. Young stars are usually excluded from planet-search programmes owing to their high-levels of chromospheric activity, although much effort is being applied to overcome this problem (e.g. Dumusque et al. 2011a,b). Finally, we can exclude a dust luminosity evolution with age in the SWDP sample, in line with the results of Trilling et al. (2008) for solar-type stars surrounded by debris discs.

\section{Conclusions}

The number of debris disc stars known to host planets has increased in the past few years by a factor of $\sim 50 \%$, particularly those associated with low-mass planets. This has motivated us to revisit the properties of these stars and to compare them with 
stars with planets, stars with debris discs, and stars with neither debris nor planets.

We have identified a transition toward higher metallicities from SWODs to SWPs. The SWDs have a metallicity distribution similar to those of SWODs, although the distribution of the first ones might be slightly shifted towards higher metallicities. The SWDPs follow the same metallicity trend as SWPs, irrespective of whether the planets are low-mass or gas giants; thus, it is the planet which reveals the metallicity of the corresponding stars. There is a high rate of incidence of multiplanet systems in SWDPs. Their innermost planets are usually cool giants, but the planets are close-in when the debris disc stars only host low-mass planets. It cannot be excluded that this latter result could be biased by the planet detection techniques. These results support the scenario of core accretion for planet formation and the previous view that the mass of solids in protoplanetary discs is the main factor determining the outcome of planet formation processes.

In addition, we have found that debris disc stars hosting cool giant planets tend to have the lowest dust luminosities, and that there is an anticorrelation between the dust luminosity and the innermost planet eccentricity. A plausible explanation of these suggested trends is provided by recent simulations of dynamical instabilities produced by eccentric giant planets. These apparent trends will likely be either confirmed or rejected by the various programmes dealing with planets and debris discs, currently being carried out with the Herschel Space Observatory, together with the expected detection of further planets, particularly lowmass planets, around the debris disc stars.

Finally, no other trend has been found relating debris disc and planet (e.g. period or semimajor axis) properties.

Acknowledgements. This work was supported by the Spanish Ministerio de Ciencia e Innovación (MICINN), Plan Nacional de Astronomía y Astrofísica, under grant AYA2008-01727. J.M. acknowledges support from the Universidad Autónoma de Madrid (Department of Theoretical Physics). We sincerely appreciate the careful reading of the manuscript and the constructive comments of an anonymous referee.

\section{Appendix A: Results of the Kolmogorov-Smirnov tests}

The Kolmogorov-Smirnov test (hereafter K-S test) is widely used to study the significance of the difference between two data samples (e.g. Peacock 1983). It is based on the maximum deviation between the empirical distribution functions of both samples

$D=\max \left|F_{1}(x)-F_{2}(x)\right|$,

where $\mathrm{F}_{1}(x)$ and $\mathrm{F}_{2}(x)$ are the empirical distribution functions of the first and second samples respectively, and are given by

$F(x)=\frac{n\left(x_{i} \leq x\right)}{N}$

The K-S test tests the null hypothesis $\mathrm{H}_{0}$ that $F_{1}(x)=F_{2}(x)$, i.e., both samples come from the same underlying continuous distribution, which is accepted if

$\max \left|F_{1}(x)-F_{2}(x)\right|<C_{\frac{\alpha}{2}, n_{1}, n_{2}}$

where $n_{1}$ and $n_{2}$ are the sizes of the samples, $\alpha$ is the confidence level, and $C$ the corresponding critical values of the $\mathrm{K}-\mathrm{S}$ distribution.

Through this paper, we perform several K-S tests between the different samples studied. Results are given in Table A.1,
Table A.1. Results of the K-S tests performed in this work.

\begin{tabular}{lccccccc}
\hline \hline Sample 1 & Sample 2 & $n_{1}$ & $n_{2}$ & $n_{\text {eff }}$ & $\mathrm{H}_{0}{ }^{\ddagger}$ & $p$ & $D$ \\
\hline SWDs $^{\star}$ & SWODs $^{\star}$ & 35 & 58 & 22 & 0 & 0.94 & 0.11 \\
SWDs & SWODs $^{\sharp}$ & 107 & 145 & 62 & 0 & 0.09 & 0.16 \\
SWDPs & SWODs & 29 & 145 & 24 & 1 & $\sim 10^{-3}$ & 0.40 \\
SWDPs & SWDs & 29 & 107 & 23 & 1 & $7 \times 10^{-3}$ & 0.34 \\
SWDPs & SWPs & 29 & 120 & 24 & 0 & 0.49 & 0.17 \\
\hline SWPs & SWODs & 120 & 145 & 66 & 1 & $\sim 10^{-11}$ & 0.43 \\
SWPs & SWDs & 120 & 107 & 57 & 1 & $\sim 10^{-10}$ & 0.44 \\
\hline
\end{tabular}

Notes. (‡) 0: Accept null hypothesis; 1: Reject null hypothesis.

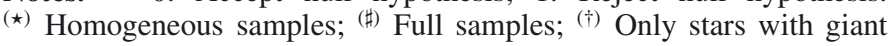
planets considered.

where the "asymptotic $p$-value" is also given. It provides an estimate of the likelihood of the null hypothesis and is reasonable accurate for samples sizes for which

$n_{\mathrm{eff}}=\frac{n_{1} \times n_{2}}{n_{1}+n_{2}} \geq 4$.

All the tests were made at a confidence level $\alpha=0.02$.

\section{References}

Allende Prieto, C., Barklem, P. S., Lambert, D. L., \& Cunha, K. 2004, A\&A, 420, 183

Aumann, H. H., Beichman, C. A., Gillett, F. C., et al. 1984, ApJ, 278, L23

Backman, D. E., \& Paresce, F. 1993, in Protostars and Planets III, ed. E. H. Levy, \& J. I. Lunine, 1253

Beichman, C. A., Bryden, G., Rieke, G. H., et al. 2005, ApJ, 622, 1160

Beichman, C. A., Bryden, G., Stapelfeldt, K. R., et al. 2006, ApJ, 652, 1674

Benítez, N., Maíz-Apellániz, J., \& Canelles, M. 2002, Phys. Rev. Lett., 88, 081101

Bryden, G., Beichman, C. A., Trilling, D. E., et al. 2006, ApJ, 636, 1098

Bryden, G., Beichman, C. A., Carpenter, J. M., et al. 2009, ApJ, 705, 1226

Chavero, C., Gómez, M., Whitney, B. A., \& Saffe, C. 2006, A\&A, 452, 921

Chen, C. H., Patten, B. M., Werner, M. W., et al. 2005, ApJ, 634, 1372

Currie, T. 2009, ApJ, 694, L171

Dodson-Robinson, S. E., Beichman, C. A., Carpenter, J. M., \& Bryden, G. 2011, AJ, 141,11

Dumusque, X., Santos, N. C., Udry, S., Lovis, C., \& Bonfils, X. 2011a, A\&A, 527, A82

Dumusque, X., Udry, S., Lovis, C., Santos, N. C., \& Monteiro, M. J. P. F. G. 2011b, A\&A, 525, A140

Fischer, D. A., \& Valenti, J. 2005, ApJ, 622, 1102

Frandsen, S., \& Lindberg, B. 1999, in Astrophysics with the NOT, ed. H. Karttunen, \& V. Piirola, 71

Garnett, D. R., \& Kobulnicky, H. A. 2000, ApJ, 532, 1192

Ghezzi, L., Cunha, K., Schuler, S. C., \& Smith, V. V. 2010a, ApJ, 725, 721

Ghezzi, L., Cunha, K., Smith, V. V., et al. 2010b, ApJ, 720, 1290

Gratton, R. G., Bonanno, G., Bruno, P., et al. 2001, Exper. Astron., 12, 107

Greaves, J. S., Fischer, D. A., \& Wyatt, M. C. 2006, MNRAS, 366, 283

Greaves, J. S., Fischer, D. A., Wyatt, M. C., Beichman, C. A., \& Bryden, G. 2007, MNRAS, 378, L1

Habing, H. J., Dominik, C., Jourdain de Muizon, M., et al. 2001, A\&A, 365, 545

Hekker, S., \& Meléndez, J. 2007, A\&A, 475, 1003

Hubickyj, O., Bodenheimer, P., \& Lissauer, J. J. 2005, Icarus, 179, 415

Ida, S., \& Lin, D. N. C. 2004, ApJ, 616, 567

Koerner, D. W., Kim, S., Trilling, D. E., et al. 2010, ApJ, 710, L26

Kóspál, Á., Ardila, D. R., Moór, A., \& Ábrahám, P. 2009, ApJ, 700, L73

Maíz-Apellániz, J. 2001, ApJ, 560, L83

Maldonado, J., Martínez-Arnáiz, R. M., Eiroa, C., Montes, D., \& Montesinos, B. 2010, A\&A, 521, A12

Mamajek, E. E., \& Hillenbrand, L. A. 2008, ApJ, 687, 1264

Martínez-Arnáiz, R., Maldonado, J., Montes, D., Eiroa, C., \& Montesinos, B. 2010, A\&A, 520, A79

Matteucci, F., \& Greggio, L. 1986, A\&A, 154, 279

Matteucci, F., \& Recchi, S. 2001, ApJ, 558, 351

Matteucci, F., Spitoni, E., Recchi, S., \& Valiante, R. 2009, A\&A, 501, 531

Mayor, M., Marmier, M., Lovis, C., et al. 2011, A\&A, submitted

[arXiv: 1109.2497]

Montes, D., López-Santiago, J., Gálvez, M. C., et al. 2001, MNRAS, 328, 45 
Moór, A., Ábrahám, P., Derekas, A., et al. 2006, ApJ, 644, 525 Moór, A., Pascucci, I., Kóspál, Á., et al. 2011, ApJS, 193, 4

Mordasini, C., Alibert, Y., \& Benz, W. 2009, A\&A, 501, 1139

Mordasini, C., Alibert, Y., Benz, W., Klahr, H., \& Henning, T. 2012, A\&A, in press, DOI: $10.1051 / 0004-6361 / 201117350$

Moro-Martín, A., Carpenter, J. M., Meyer, M. R., et al. 2007, ApJ, 658, 1312

Nordström, B., Mayor, M., Andersen, J., et al. 2004, A\&A, 418, 989

Pasquini, L., Döllinger, M. P., Weiss, A., et al. 2007, A\&A, 473, 979

Peacock, J. A. 1983, MNRAS, 202, 615

Pfeiffer, M. J., Frank, C., Baumueller, D., Fuhrmann, K., \& Gehren, T. 1998, A\&AS, 130, 381

Plavchan, P., Werner, M. W., Chen, C. H., et al. 2009, ApJ, 698, 1068

Pollack, J. B., Hubickyj, O., Bodenheimer, P., et al. 1996, Icarus, 124, 62

Raymond, S. N., Armitage, P. J., Moro-Martín, A., et al. 2011, A\&A, 530, A62

Rhee, J. H., Song, I., Zuckerman, B., \& McElwain, M. 2007, ApJ, 660, 1556

Santos, N. C., Israelian, G., \& Mayor, M. 2004, A\&A, 415, 1153
Smith, P. S., Hines, D. C., Low, F. J., et al. 2006, ApJ, 644, L125

Sousa, S. G., Santos, N. C., Mayor, M., et al. 2008, A\&A, 487, 373

Sousa, S. G., Santos, N. C., Israelian, G., Mayor, M., \& Udry, S. 2011, A\&A, 533, A141

Spangler, C., Sargent, A. I., Silverstone, M. D., Becklin, E. E., \& Zuckerman, B. 2001, ApJ, 555, 932

Takeda, Y., Ohkubo, M., \& Sadakane, K. 2002, PASJ, 54, 451

Takeda, Y., Sato, B., Kambe, E., et al. 2005, PASJ, 57, 109

Tanner, A., Beichman, C., Bryden, G., Lisse, C., \& Lawler, S. 2009, ApJ, 704, 109

Timmes, F. X., Woosley, S. E., \& Weaver, T. A. 1995, ApJS, 98, 617

Trilling, D. E., Stansberry, J. A., Stapelfeldt, K. R., et al. 2007, ApJ, 658, 1289

Trilling, D. E., Bryden, G., Beichman, C. A., et al. 2008, ApJ, 674, 1086

Valenti, J. A., \& Fischer, D. A. 2005, ApJS, 159, 141

van Leeuwen, F. V. 2007, Hipparcos, the New Reduction of the Raw Data XXXII, Hardcover, Astrophys. Space Sci. Lib., 350

Wright, J. T., Upadhyay, S., Marcy, G. W., et al. 2009, ApJ, 693, 1084 